\title{
SITUACIONES SOFÍSTICAS EN EL APRENDIZAJE DE LA FÍSICA. ESTRATEGIAS PARA SU PUESTA EN PRÁCTICA EN EL AULA
}

\author{
Antonio García Carmona \\ Área de Ciencias (Física y Química), Colegio Luisa de Marillac, España
}

\section{INTRODUCCIÓN}

Una de las prácticas más utilizadas en la enseñanza de la Física es proponer relaciones de problemas tipo, basados en ejercicios de aplicación, que requieren la utilización de reglas y algoritmos, que son aprendidos y aplicados por los alumnos de forma automática (Neto y Valente, 2001). Esta práctica didáctica contribuye a que los alumnos adquieran una habilidad rutinaria, también denominada metodología de la superficialidad (Buteler, 2001), con la que llegan a resolver los problemas de forma mecánica y acrítica (Gil et al, 1991; Gil y Guzmán, 1993; Perales, 2003). Con lo cual, no se profundiza en el contenido conceptual del problema, ya que los alumnos, al encontrar un patrón de cuestiones ya establecido, no conciben cómo se debe plantear un problema de investigación en Física. Por este motivo, cuando se introducen pequeñas variaciones en un problema "conocido" por los alumnos, suelen incurrir en resultados erróneos.

En la mayoría de las ocasiones, cuando el alumno se enfrenta a un problema en clase, lo analiza de forma superficial sin recapacitar si los datos ofrecidos son o no razonables, o si el resultado al que se llega es congruente o no (García Carmona, 2004). En cambio, suele centrar su atención en detectar qué "tipo de problema" tiene delante, con el fin de aplicar de manera mecánica, y a veces indiscriminada, las ecuaciones que tiene en su cabeza. Una estrategia para potenciar la utilidad didáctica de la resolución de problemas consiste en analizar y resolver situaciones físicas presentadas como sofismas (García Carmona, 2001). Se trata de plantear cuestiones o problemas en los que se ofrecen datos incompatibles, o se introducen ciertos errores de procedimiento, de forma intencionada, que dan lugar a un resultado incongruente. El propósito es que los alumnos detecten y analicen esas incoherencias, al tiempo que desarrollen una serie de actitudes y destrezas, propias del quehacer científico, esenciales en el aprendizaje de la Física (García García, 2000; Vásquez et al, 2004).

\section{ORIGEN Y SIGNIFICADO DE UN SOFISMA CIENTÍFICO}

El desarrollo del intelecto humano se ha fundamentado en la búsqueda de métodos orientados a establecer una línea de razonamiento que nos permitan comprender los fenómenos que nos rodean (García Carmona, 2002). Así, por ejemplo, las Ciencias y las Matemáticas utilizan el método axiomático, que consiste en establecer una serie de enunciados (también llamados axiomas o principios) a los que se les aplica un conjunto de reglas, que pueden ser aritméticas, lógicas, o la que convenga en cada caso. Estos principios pueden ser evidentes o simplemente aceptados para los fines del razonamiento posterior. En Física encontramos multitud de principios o axiomas científicos, como, por ejemplo, el de conservación de la energía, el de Pascal, el de Arquímedes, el de Inercia, etc. 
Ya en la antigua Grecia eran conocidos los métodos de razonamiento inductivo y deductivo. El primero consiste en un procedimiento empírico basado en la observación, cuya aplicación orienta el proceso de razonamiento desde lo particular a lo general. Por el contrario, el método deductivo se basa en premisas lógicas que son aplicadas siguiendo un razonamiento de lo general a lo particular. Para ello, parte de ciertas premisas (hipótesis), que han de ser contrastadas mediante procedimientos empíricos; lo que conocemos como método científico.

Sin embargo, antes del establecimiento de los métodos de razonamiento anteriores, desarrollados por filósofos como Sócrates, Platón y Aristóteles, en Grecia existían los sofistas, también llamados 'maestros de la sabiduría' (Padrón, 2000). Eran verdaderos titiriteros de la palabra y hábiles en la construcción de discursos llenos de grandilocuencia y de estratagemas argumentales. Partiendo de la debilidad intelectual de las masas, los sofistas ${ }^{1}$ usaban su oratoria, mediante el uso de argumentos incorrectos, con una finalidad persuasoria de dar a entender que poseían un conocimiento pleno del mundo. Desde entonces, se denomina sofisma ${ }^{2}$ a un argumento basado en razonamientos falsos, que son introducidos intencionadamente, con el propósito de confundir a alguien cuando se le quiere convencer de algo (Langue, 1984).

Aun cuando existen numerosos tipos de sofismas, todos ellos emplean argumentos erróneos basados en: a) afirmaciones falsas como si fuesen afirmaciones verdaderas, y b) procedimientos de inferencia incorrectos. Obviamente pueden ocurrir las dos clases de defectos en una misma argumentación sofística. Con el fin de ilustrar lo que acabamos de decir, exponemos a continuación algunos ejemplos de sofismas clásicos de índole científica.

- Sofisma de petición de principio. Consiste en admitir desde un principio aquello que está precisamente en cuestión y hay que demostrar. Por ejemplo: «Todo efecto tiene una causa. El Universo es un efecto, por tanto, el Universo tiene una causa». El argumento parece correcto, pero se observa que al poner la segunda premisa se comete petición de principio, dado que se afirma la conclusión que se pretende demostrar, porque al decir "el Universo es un efecto" se está afirmando que "el Universo ha sido causado".

- Sofisma "ad ignorantiam" (argumento de la ignorancia ajena). Ocurre cuando se pretende dar por probada una tesis partiendo de un hecho del cual no se ha podido probar la tesis contraria. Por ejemplo: «Nadie ha demostrado que no existan los seres extraterrestres. Por lo tanto, hay vida en otros planetas».

- Sofisma de falsa generalización. Se parte de la afirmación de que una propiedad es poseída por varios individuos de un conjunto, y a partir de ello se concluye que todos los elementos de ese conjunto poseen tal propiedad, aun cuando no se tenga certeza de ello. Por ejemplo: «El neón, el argón y el criptón son gases nobles, que tienen ocho electrones de valencia. Por tanto, los elementos de la tabla periódica que no poseen ocho electrones en su capa de valencia, no

\footnotetext{
${ }^{1}$ Con Sócrates, Platón y Aristóteles, los sofistas fueron perdiendo credibilidad, de manera progresiva, principalmente por su creciente malabarismo retórico y por su incapacidad de dar una explicación del mundo.

${ }^{2}$ Para algunos tratadistas, el término sofisma es sinónimo de falacia.
} 
son gases nobles». Sin embargo, es bien sabido que el helio es un gas noble que posee sólo dos electrones de valencia.

- Sofisma de analogía. Se parte de la premisa de que dos cosas coinciden en algunos aspectos probados, de modo que si el aspecto ha sido comprobado en sólo una de ellas, también se da como válido en la otra. Este tipo de razonamiento se denomina "razonamiento por analogía" y es válido cuando la conclusión se postula como probable; pero si se concibe como cierta, es un sofisma. Por ejemplo: “Marte tiene un movimiento de rotación sobre su eje, como la Tierra. Marte tiene atmósfera, como la Tierra. Marte tiene agua en su superficie, como la Tierra. Marte tiene estaciones, como la Tierra. Por tanto, Marte tiene seres vivos, como la Tierra».

\section{MARCO DIDÁCTICO PARA LA PUESTA EN PRÁCTICA DE SITUACIONES SOFÍSTICAS}

Antes de presentar la propuesta, conviene establecer el marco didáctico en el que se han de poner en práctica las situaciones sofísticas. En sintonía con el paradigma del constructivismo, la resolución de situaciones sofísticas en el aula de Física debe plantearse de manera que propicien el cambio conceptual pertinente, con vistas a que los alumnos logren un aprendizaje significativo (Marín, 2003; Moreira y Greca, 2003). Esto requiere de un proceso activo de relación, diferenciación y reconciliación integradora de lo aprendido con los conceptos que ya existen en la mente de quien aprende (Rosado y Ayensa, 1999; Barboza, 2004).

En el marco del constructivismo, el planteamiento de situaciones sofísticas en Física tiene como propósito una planificación del aprendizaje como investigación (Martín, Gómez y Gutiérrez, 2000; Rosado y Ayensa, 1999). De modo que la puesta en práctica en el aula estará fundamentada en el modelo de aprendizaje basado en la resolución de problemas (Campanario y Moya, 1999; Perales, 2003). Siguiendo las directrices establecidas por Gil y Guzmán (1993), a la hora de plantear situaciones sofísticas en el aula de Física se han de tener en cuenta las siguientes pautas:

- Considerar cuál puede ser la utilidad de la situación sofística abordada, en función de los intereses del proceso de enseñanza/aprendizaje.

- Comenzar por un estudio cualitativo de la situación, intentando acotar y definir, de manera precisa, el problema, explicitando las condiciones que se consideran esenciales.

- Emitir hipótesis fundadas sobre los factores intervinientes en el fenómeno estudiado.

- Elaborar y explicitar posibles estrategias de resolución antes de proceder a ésta, evitando el puro ensayo y error. Buscar distintas vías de resolución para posibilitar la contrastación de los resultados obtenidos y mostrar la coherencia del cuerpo de conocimientos del que se dispone.

- Realizar la resolución verbalizando al máximo, fundamentando lo que se hace y evitando operativismos carentes de significado físico.

- Analizar cuidadosamente los resultados a la luz de las hipótesis elaboradas y, en particular, de los casos límite considerados.

Por otra parte, la estrategia metodológica para la puesta en práctica de las situaciones sofísticas estará fundamentada en lo que sigue: 
- Las situaciones sofísticas propuestas deben ser creativas y presentadas con carácter lúdico e imaginativo, a fin de atraer la atención del alumno como elemento de motivación. Asimismo, se establecerá correspondencia entre las situaciones sofísticas y los conceptos a enseñar, con el propósito de que la resolución permita la construcción de los conceptos.

- Se debe propiciar un clima de aula ameno y estimulante. Para ello, se fomentará un ambiente de seguridad cognitiva para el alumno, en el que se apoye la crítica, la reflexión y la expresión, y en el que se conciba el error como una oportunidad, más que como una dificultad (Meneses, 1999). Esto se llevará a cabo del modo siguiente:

- Fomento de la comunicación entre los alumnos, con objeto de que argumenten sus ideas y debatan sobre las mismas. Ello propiciará el desarrollo de la imaginación y de la percepción, además de facilitar los procesos de autoevaluación (Ayensa, 2001). En este sentido, serán importantes las puestas en común, orientadas a confrontar ideas y consensuar conclusiones.

- Organización de los alumnos en grupos de trabajo (Cordero et al, 2002; Rosado, Gómez e Insausti, 2001), ya que la interacción entre individuos de nivel cognitivo similar favorece el aprendizaje.

- Establecimiento de las condiciones espacio-temporales adecuadas, que impliquen la flexibilización del tiempo, la asignación de espacios físicos y de recursos para el trabajo autónomo del alumno (García García, 2000).

- Utilización de un sistema de autorregulación del aprendizaje (Ayensa, 2001), como guía metacognitiva a través de la cual los alumnos regulen sus propio aprendizaje (Campanario y Moya, 1999). El sistema de autorregulación se desarrollará mediante los siguientes instrumentos:

- Fichas de trabajo. Serán la guía de trabajo en las que se incluyen las situaciones sofísticas a estudiar y las herramientas heurísticas a emplear en su resolución. La información recogida en estas fichas puede ser de tipo cualitativo o cuantitativo según la naturaleza de la situación sofística que se pretenda resolver.

- Cuadernos de trabajo. Se emplearán para registrar los procesos y actos cognitivos llevados a cabo durante la resolución de las situaciones sofísticas planteadas. Los alumnos consignarán las soluciones preliminares y finales, los pasos, algoritmos y cálculos realizados, o pensados pero no ejecutados. También reflejarán las propuestas de corrección y las reflexiones globales sobre los aspectos de mayor interés y sobre los aportes metodológicos y conceptuales, obtenidos a través del proceso de resolución.

\section{PROPUESTA DE SITUACIONES SOFÍSTICAS EN FÍSICA}

A continuación se proponen diversas situaciones sofísticas sobre Física, que han de plantearse en el marco didáctico y metodológico expuesto. Existen muchos tipos de sofismas, si bien, por razones de espacio, en este artículo sólo proponemos algunos ejemplos, que pueden ser aplicados en el aula de Física 
a partir de Bachillerato (16-18 años). En función del modo en que se insertan los datos o razonamientos erróneos, distinguiremos tres tipos de situaciones sofísticas:

- Situaciones sofísticas con incongruencias en el enunciado. Aquí planteamos dos modalidades:

a) Introducción de datos incorrectos o incoherentes en el enunciado, que llevan a resultados distintos de un problema cerrado, según el método empleado, pese a que estos son correctos.

b) Inducción al empleo de conceptos y leyes físicas, cuya utilización es improcedente por la fenomenología del problema, con idea de que el resultado final obtenido sea absurdo o incorrecto.

- Situaciones sofísticas en las que los errores son introducidos durante su resolución. Son problemas en los que se emplean argumentos físicos erróneos (leyes o principios físicos improcedentes) a la hora de plantear su resolución.

- Situaciones sofísticas que presentan aparentes incongruencias en los resultados. Son problemas cuyos resultados aparentan ser erróneos o paradójicos para el alumno, pero que son realmente correctos. Se aprovecha la tendencia habitual del alumno de interpretar los fenómenos desde su propia intuición, en lugar de hacerlo desde una perspectiva científica.

Conviene precisar que hemos optado por mostrar ejemplos que suelen ser conocidos por el profesorado de Física, dado que nuestra atención se centra en ilustrar la utilidad didáctica de este recurso y no en valorar las propias cuestiones. Entendemos, en este sentido, que la propuesta debe ser considerada como un referente que sirva a cada profesor, de acuerdo con las necesidades de su alumnado, a plantear las cuestiones más apropiadas en cada caso. Asimismo, las situaciones sofísticas que se presentan son susceptibles de cuantas modificaciones se estimen oportunas a fin de mejorar su aprovechamiento didáctico.

\subsection{Situación sofística con incongruencias en el enunciado}

En la mayoría de las ocasiones, cuando el alumno se enfrenta a un problema de clase, lo analiza de forma superficial sin pararse a ver si los datos ofrecidos son o no razonables. Centra su atención en detectar qué tipo de problema tiene delante, con idea de aplicar de manera mecánica todas las ecuaciones que conoce sobre el tema. Un buen modo de mostrar a los alumnos la importancia de comprender adecuadamente el enunciado de un problema, consiste en plantearlo con datos incorrectos o incoherentes; o bien, plantearlo con un enunciado que les incite a realizar un planteamiento incorrecto del problema. De ambas formas, se les conducirá a un resultado absurdo, que les hará reflexionar sobre ello con mayor interés que con problemas enunciados correctamente. llustremos esto con los dos ejemplos que siguen.

\section{a) Ejemplo de problema con datos incongruentes en el enunciado}

A un cuerpo de $1 \mathrm{Kg}$ de masa se le aplicó una fuerza que le provocó un cambio de velocidad de 10 $\mathrm{m} / \mathrm{s}$ a $30 \mathrm{~m} / \mathrm{s}$ en $5 \mathrm{~s}$, recorriendo un trayecto recto de $25 \mathrm{~m}$. Despreciando todo tipo de rozamientos, determina el valor de dicha velocidad.

Resolución. El problema puede ser resuelto de dos maneras diferentes; bien a través de la $2^{z}$ ley de Newton, o bien mediante un balance energético, empleando el teorema de las fuerzas vivas. 
Por la primera forma:

$F=m a=m\left(v_{2}-v_{1}\right) / t=1 \mathrm{Kg}(30 \mathrm{~m} / \mathrm{s}-10 \mathrm{~m} / \mathrm{s}) / 5 \mathrm{~s}=4 \mathrm{~N}$

Por la segunda forma:

$F \ddot{A} s=1 / 2 m\left(v_{2}\right)^{2}-1 / 2 m\left(v_{1}\right)^{2}$

y despejando la fuerza se tiene:

$\left.F=1 / 2(m / A ̈ s)\left[\left(v_{2}\right)^{2}-\left(v_{1}\right)^{2}\right]=1 / k 1 \mathrm{Kg} / 25 \mathrm{~m}\right)\left[900 \mathrm{~m}^{2} / \mathrm{s}^{2}-100 \mathrm{~m}^{2} / \mathrm{s}^{2}\right]=16 \mathrm{~N}$

¿Por qué tiene el problema dos soluciones distintas? ¿Acaso pueden ser a la vez correctas dos soluciones diferentes?

Obviamente, ambas soluciones del problema son irrefutables en cuanto a que las ecuaciones utilizadas y los cálculos realizados son correctos. Esto, por tanto, debe inducir a los alumnos a pensar que el error no se encuentra en la resolución sino en el enunciado del problema, que ofrece una información excesiva e incoherente. En efecto, no es posible que un cuerpo pase de tener $10 \mathrm{~m} / \mathrm{s}$ a $30 \mathrm{~m} / \mathrm{s}$ durante $5 \mathrm{~s}$, y recorra un espacio de $25 \mathrm{~m}$. Para esos valores de velocidad y tiempo, el espacio recorrido debe ser $100 \mathrm{~m}$. De manera que el dato del espacio es redundante e incorrecto. También es posible que sean correctos los datos de las velocidades y el espacio y el referido al tiempo no. De cualquiera de las maneras, existe una incongruencia en los datos, que da lugar a este interesante "misterio", interesante para ser discutido en clase.

\section{b) Ejemplo de inducción a un planteamiento incorrecto a partir del enunciado}

Se tienen dos bloques de masas $M_{1}$ y $M_{2}$ en reposo y situados sobre una superficie horizontal perfectamente lisa (figura 1). A continuación se aplica una fuerza $F$ al bloque de la izquierda, que a través de éste, actuará sobre el bloque de la derecha. Determina con qué aceleración se moverá el bloque de la izquierda.

Figura 1.

Sistema de dos bloques situados en una superficie horizontal sin fricción.

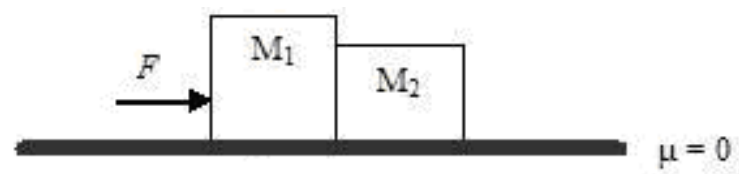

Resolución. Con base en la $3^{a}$ ley de Newton (o principio de acción y reacción), si sobre $M_{2}$ se ejerce la fuerza $F$ a través de $M_{1}, M_{2}$ ejercerá la misma fuerza y de sentido contrario, sobre $M_{1}$. De modo que si hacemos un balance de fuerzas sobre $M_{1}$, tenemos que la suma de todas las fuerzas es igual a cero:

Dirección vertical: $N-m g=0$

Dirección horizontal: $F-F_{\text {reacc. }}=F-F=0=m \cdot a ; a=0$

Con este resultado, tenemos que el bloque $M_{1}$ jamás llegará a moverse por muy grande que sea la fuerza que le apliquemos. ¿No es este un resultado muy extraño?

Al igual que en el ejemplo anterior, el error de razonamiento tiene su origen en el enunciado del problema cuando se afirma: "...se aplica una fuerza $F$ al bloque de la izquierda, que a través de éste, actuará sobre el bloque de la derecha". En efecto, no es cierto que la fuerza $F$, aplicada al bloque de la izquierda, actúe también sobre el de la derecha tal como se indica. Sobre $M_{2}$ actuará una fuerza $F^{\prime} \neq F$, a consecuencia de su contacto con $M_{1}$, el cual, a su vez, recibirá la misma fuerza $F$, pero en sentido contrario, 
de acuerdo con el principio de acción y reacción. De manera que si aplicamos la $\mathfrak{2}$ ley de Newton a cada cuerpo en la dirección del movimiento, tenemos que las aceleraciones de cada uno son: $a_{1}=\frac{F-F^{\prime}}{M_{1}}, a_{2}$ $=\frac{F^{\prime}}{M_{2}} ;$ y como ambos se mueven unidos, la aceleración de ambos es la misma. Con lo cual, tenemos que $\frac{F^{\prime}}{M_{2}}=\frac{F-F^{\prime}}{M_{1}}$, de donde se concluye que $F^{\prime}=\frac{M_{2}}{M_{1}+M_{2}} F$; es decir, $F^{\prime}<F$, por tanto, $M_{1}$ siempre podrá moverse, sea cual sea el valor de $\mathrm{F}$, al no existir rozamiento.

Es otro ejemplo en el cual, si no se presta la atención suficiente y se analiza detenidamente el enunciado, se puede incurrir en el error conceptual que acabamos de comentar: "la transmisión de fuerzas a través de los cuerpos". Una vez detectado dicho error, será interesante pedir a los alumnos que reformulen el problema de manera correcta.

\subsection{Sofismas con incongruencias introducidas durante el razonamiento}

En la enseñanza de la Física, es habitual plantear desarrollos teóricos orientados a una determinada ecuación o ley física. Bien los realiza el profesor en clase, de manera magistral, o bien se proponen a los alumnos como ejercicios, a fin de que desarrollen ciertas destrezas en la manipulación de ecuaciones. En estos desarrollos, se han de emplear fundamentos y principios físicos, así como ciertas herramientas matemáticas, que combinados de una manera adecuada, nos llevan a una expresión final. Son, por tanto, un escenario ideal para trabajar la capacidad de análisis y de razonamiento, dado que son muy susceptibles de que se les puedan introducir errores sutilmente, que conduzcan a un resultado incoherente. El que sigue es un ejemplo en el que se introduce un error de tipo físico durante el desarrollo de la cuestión.

Determinación de la fórmula del periodo de un péndulo simple.

Resolución. Para ello, consideraremos oscilaciones pequeñas respecto de la posición de equilibrio y la masa del hilo despreciable. En la figura 2, representamos un esquema con la descomposición de las fuerzas que actúan sobre el cuerpo.

Aplicando la $2^{2}$ ley de Newton para las direcciones normal y tangencial del movimiento de la bola, resulta:

$$
\begin{aligned}
& T-m g \text { cosè }=0 \\
& -m g \text { senè }=m a
\end{aligned}
$$

Dado que las oscilaciones son pequeñas, podemos aproximar senè $\approx$ è; además, la longitud del arco, s, está relacionada con el ángulo por $s=$ è l. Con esto, la aceleración vendrá dada como:

$$
a=-g \grave{e}=-g s / l
$$

Por otro lado, a partir de la ecuación espacio-temporal de un movimiento uniformemente acelerado, el tiempo en recorrer el arco s se puede expresar como sigue:

$$
t=(2 s / a) 1 / 2
$$

De modo que la fórmula anterior se puede expresar de esta otra forma: 


$$
t=(2 / / g) 1 / 2
$$

Teniendo en cuenta que el valor del periodo de péndulo será 4 veces el tiempo empleado en el trayecto $A B$, resulta:

$$
T=4(2 / / g) 1 / 2
$$

Sin embargo, la fórmula para el periodo, mostrada en los libros de texto, es $T=2 \delta(\mathrm{l} / \mathrm{g})^{1 / 2}$. ¿Dónde está el error?

Figura 2

Péndulo simple

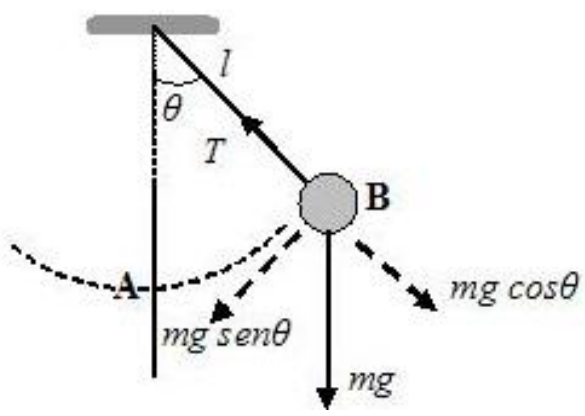

Evidentemente existe un error de tipo conceptual en el desarrollo. Hemos asumido que se trata de un movimiento uniformemente acelerado (particularizando el valor de la aceleración al instante de amplitud máxima) cuando no es cierto. La aceleración de la bola, según la expresión (3), es directamente proporcional a la amplitud del ángulo de oscilación, è, del péndulo; de manera que ésta variará a lo largo del movimiento. La introducción intencionada de este error viene a colación de la limitación que suele hacerse -principalmente en los niveles de enseñanza no universitarios- del estudio de movimientos a uniformemente acelerados. Por tanto, es fácil que nuestro desarrollo pase desapercibido en una clase de Física de Bachillerato, si no se alerta de la existencia de un error tal como hemos hecho al final del mismo.

La resolución correcta parte de expresar la aceleración, dada por la expresión (3), como la segunda derivada temporal de la posición:

$$
\text { -g. } \frac{s}{l}=\frac{d^{2} s}{d t^{2}}
$$

Puesto que las oscilaciones son pequeñas, tenemos un movimiento armónico, ya que la aceleración es directamente proporcional al desplazamiento $s$. De modo que la ecuación diferencial anterior se puede expresar como:

$$
-\omega^{2} s=\frac{d^{2} s}{d t},
$$

Siendo $w=(g / l)^{1 / 2}$, la frecuencia angular de oscilación del péndulo. Puesto que la relación del periodo con dicha frecuencia viene dada por la expresión $T=2 \delta / w$, llegamos a que el periodo de un péndulo simple es:

$$
\mathrm{T}=2 \circlearrowright(\mathrm{I} / \mathrm{g}) 1 / 2
$$

\subsection{Problemas con aparentes incongruencias en el resultado}


A veces, tanto alumnos como profesores, nos apresuramos a desestimar -0 , al menos, poner en dudas - los resultados de un problema porque, en una primera revisión, no nos cuadran (Gil et al, 1991). Esto responde, como señalábamos al principio, a un mecanicismo del que nos hemos ido forjando a causa de la superficial y rutinaria forma de plantear y resolver problemas en clase.

Un científico, sin embargo, no suele apresurarse a desestimar un resultado sin antes haber revisado todas las posibilidades existentes; más aún cuando los procedimientos empleados han sido los correctos. En multitud de ocasiones, la solución no es la que nos dicta nuestro sentido común, sino que es preciso hacer una reflexión coherente con el razonamiento científico para encontrarla. Una buen modo de desarrollar este procedimiento es a través del planteamiento de cuestiones cuyos resultados parecen equivocados, pero que, sin embargo, son totalmente correctos. Como ejemplo, analicemos la situación que sigue.

A Pedro, un alumno de Bachillerato, le preguntaron en un examen de Física lo siguiente: "Como es sabido, el punto de ebullición del aceite de oliva es más alto que el punto de fusión del estaño. Explica, entonces, ¿por qué se pueden freír alimentos en aceite de oliva utilizando una cacerola estañada?»

Se trata de un sofisma de antecedente incompleto, es decir, que intenta centrar la atención sólo en una parte del fenómeno, omitiendo otro aspecto relevante, que es decisivo para su explicación. En efecto, el enunciado trata de centrar toda la atención en el aceite y el estaño; sin embargo, no hace énfasis en las propiedades de los alimentos. Cuando se fríe un alimento, no hierve el aceite sino el agua contenida en los alimentos; de modo que, mientras no se haya evaporado toda el agua, la temperatura no sobrepasa los $100^{\circ} \mathrm{C}$ (temperatura inferior al punto de fusión del estaño y del aceite de oliva). Por este motivo, es posible hervir agua en un cucurucho de papel.

\section{CONCLUSIONES FINALES}

La reflexión sobre situaciones sofísticas en la enseñanza de la Física, coadyuva a potenciar la capacidad de razonamiento de los alumnos, puesto que se vuelven más cautos y reflexivos a la hora de afrontar cualquier tipo de cuestión o problema. Asimismo, permite afianzar mejor los conceptos manejados, puesto que se corrigen muchas de las lagunas cognitivas que perduran después del aprendizaje. Obviamente, para que este recurso surta los efectos didácticos deseados, es preciso que los alumnos trabajen previamente en el aula los conceptos que intervienen en las situaciones que se planteen.

El planteamiento se situaciones sofísticas se aconseja a partir de Bachillerato (16-18 años); no obstante, se pueden hacer las adaptaciones oportunas para cursos inferiores, si bien ello debe surgir de la experiencia de cada profesor y de las necesidades de su alumnado.

El planteamiento de situaciones sofísticas se presenta como un recurso didáctico que puede fomentar actitudes y destrezas científicas, con vistas a hacer del aprendizaje de la Física algo más atractivo y sugerente, al tiempo que mejorar el rendimiento de los alumnos y la labor del profesorado.

\section{REFERENCIAS BIBLIOGRÁFICAS}

AYENSA, J. M. (2001). Instrumentos de regulación y modelo de evaluación en el aula de Física. Tesis Doctoral. Madrid: UNED. 
BarbozA, L. (2004). Concepciones epistemológicas en la enseñanza. Contexto Educativo, 30. Disponible en línea en: http://www.contexto-educativo.com.ar/2004/1/nota-05.htm.

BUTELER, L. et al (2001). La resolución de problemas en Física y su representación: un estudio en la escuela media. Enseñanza de las Ciencias, 19 (2), 285-295.

Campanario, J. M. y Moya, A. (1999). ¿Cómo enseñar Ciencias? Principales tendencias y propuestas. Enseñanza de las ciencias, 17 (2), pp. 179-192.

Cordero, S. et al (2002). ¿Y si trabajan en grupo...? Interacciones entre alumnos, procesos sociales y cognitivos en clases universitarias de Física. Enseñanza de las Ciencias, 20 (3), pp. 427-441.

García Carmona, A. (2001). El planteamiento de sofismas y paradojas como recurso didáctico en la enseñanza de la Física. Revista Española de Física, 15 (3), pp. 34-36.

García Carmona, A. (2002). Ciencia y pensamiento ilustrado. Red Científica: Ciencia, Tecnología y Pensamiento. Disponible en línea en: http:// www.redcientifica.com/doc/doc200211220001.html.

García Carmona, A. (2004). Detección de errores e incongruencias en problemas de Física: su utilidad didáctica. Alambique: Didáctica de las Ciencias Experimentales (Aceptado para su publicación).

García García, J. J. (2000). La solución de situaciones problemáticas: una estrategia didáctica para la enseñanza de la Química. Enseñanza de las Ciencias, 18 (1), pp. 113-129.

GIL, D. et al (1991). La enseñanza de las Ciencias en la Educación Secundaria. Barcelona: ICE Universidad de Barcelona / Horsori.

GIL, D. y Guzmán, M. (1993). Enseñanza de las Ciencias y la Matemática. Tendencias e Innovaciones. Biblioteca Virtual: Organización de Estados Iberoamericanos (OEI) para la Educación, la Ciencia y la Cultura / Editorial Popular.

LANGUe, V. (1984). Paradojas y sofismas físicos. Moscú: Mir.

Marín, N. (2003). Visión constructivista dinámica para la enseñanza de las Ciencias. Enseñanza de las Ciencias, ํㅡ extra, pp. 43-55.

Martín, M. J., Gómez, M. A. y GutiérRez, M. S. (2000). La Física y la Química en Secundaria. Madrid: Narcea.

MENESES, J. A. (1999). El aprendizaje del electromagnetismo en la Universidad. Ensayo de una metodología constructivista. Burgos: Universidad de Burgos.

MoReIRA, M. A. y GRECA, I. (2003). Cambio conceptual: análisis crítico y propuestas a la luz de la teoría del aprendizaje significativo. Ciência \& Educação, 9 (2), pp. 301-315.

Neto, A. y Valente, M. O. (2001). Disonancias pedagógicas en la resolución de problemas de Física: una propuesta para su superación de raíz vygotskiana. Enseñanza de las Ciencias, 19 (1), 21-30.

Padrón, J. (2000). La Neosofística y los nuevos sofismas. Cinta de Moebio, 8. Facultad de Ciencias Sociales, Universidad de Chile. Disponible en línea en: http://rehue.csociales.uchile.cl/publicaciones/moebio/08/frames04.htm. 
Perales, F. J. (2003). La resolución de problemas de Física. Análisis crítico y propuestas alternativas. México: Memorias del XI Taller Internacional "Nuevas Tendencias en la Enseñanza de la Física”, pp. 29-34.

Rosado, L. y Ayensa, J. M. (1999). Enseñanza de la Física en el nuevo Sistema Educativo. Bases didácticas y nuevos medios tecnológicos en la ESO y Bachillerato. Madrid: UNED.

Rosado, L., Gómez, J. A. e InSAustI, M. J. (2001). Una epistemología centrada en el alumno frente a la concepción habitual del ciclo enseñanza/aprendizaje en Ciencias: reflexivo/cooperativo. En Rosado, L. y Cols. (Eds.), Didáctica de la Física y sus Nuevas Tendencias (Manual de 2001), pp. 516-560. Madrid: UNED.

VÁsqueZ, S. et al (2004). Planteo de situaciones problemáticas como estrategia integradora en la enseñanza de las Ciencias y la Tecnología. Revista Electrónica de Enseñanza de las Ciencias, 3 (1). Disponible en línea en: http://www.saum.uvigo.es/reec/volumenes/volumen3/Numero1/Art4.pdf. 


\title{
Contactar
}

Revista lberoamericana de Educación

\author{
Principal OEI
}

